\title{
Misspelling Oblivious Word Embeddings
}

\author{
Bora Edizel \\ Facebook AI, London \\ edizel@fb.edu
}

\author{
Rui Ferreira* \\ Spotify, London \\ ruifespotify.com
}

\author{
Aleksandra Piktus \\ Facebook AI, London \\ piktus@fb.com
}

\author{
Edouard Grave \\ Facebook AI, Paris \\ egraveefb.com
}

\author{
Piotr Bojanowski \\ Facebook AI, Paris \\ bojanowski@fb.com
}

\author{
Fabrizio Silvestri \\ Facebook AI, London \\ fsilvestri@fb.com
}

\begin{abstract}
In this paper we present a method to learn word embeddings that are resilient to misspellings. Existing word embeddings have limited applicability to malformed texts, which contain a non-negligible amount of outof-vocabulary words. We propose a method combining FastText with subwords and a supervised task of learning misspelling patterns. In our method, misspellings of each word are embedded close to their correct variants. We train these embeddings on a new dataset we are releasing publicly. Finally, we experimentally show the advantages of this approach on both intrinsic and extrinsic NLP tasks using public test sets.
\end{abstract}

\section{Introduction}

Word embeddings constitute a building block of many practical applications across NLP and related disciplines. Techniques such as Word2 Vec (Mikolov et al., 2013a,b) and GloVe (Pennington et al., 2014) have been extensively used in practice. One of their drawbacks, however, is that they cannot provide embeddings for words that have not been observed at training time, i.e. Out-OfVocabulary (OOV) words. In real-world tasks, the input text is often generated by people and misspellings, a common source of OOV words, are frequent (e.g. (Cucerzan and Brill, 2004) report that misspellings appear in up to $15 \%$ of web search queries). As a consequence, the quality of downstream applications of word embeddings in real-world scenarios diminishes.

Simply allowing the inclusion of misspellings into corpora and vocabularies in existing methodologies might not provide satisfactory results. The sparsity of misspellings would most likely prevent

* This work was carried out when the author was working as an employee at Facebook London. their embeddings from demonstrating any interesting properties. Trying to balance the representation of misspellings with the representation of correctly spelled variants in training data by artificially introducing misspelled variants for every word in the corpus would on the other hand cause up to an exponential growth in the size of the training data, making training of the models infeasible.

To address this deficiency, we propose Misspelling Oblivious (word) Embeddings (MOE), a new model combining FastText (Bojanowski et al., 2017) with a supervised task which embeds misspellings close to their correct variants. We carry out experiments on well established tasks and on their variants adapted to the misspellings problem. We also propose new methods of evaluating embeddings specifically designed to capture their quality on misspelled words. We train MOE embeddings on a new dataset we are releasing publicly. Finally, we experimentally show the advantages of this approach on both intrinsic and extrinsic NLP tasks using public test sets. Summarizing, we propose the following contributions:

- a novel problem and a non-trivial solution to building word embeddings resistant to misspellings;

- a novel evaluation method specifically suitable for evaluating the effectiveness of MOE;

- a dataset of misspellings ${ }^{1}$ to train MOE.

The reminder of this paper is structured as follows. Section 2 gives an overview of the word embeddings literature. In Section 3.1 we introduce Word2Vec and FastText models. We introduce the MOE model in Section 3.2. Section 4 contains the descriptions of datasets we trained on and section 5 contains the description of experiments we conducted and their results. In Section 6 we present our conclusions and plans for further research.

\footnotetext{
${ }^{1}$ https://bitbucket.org/bedizel/moe
} 


\section{Related Work}

One of the first works to introduce the concept of a distributed representation for symbolic data was (Hinton, 1986). Later, the Information Retrieval community proposed techniques of embedding words into a vector space. Latent Semantic Indexing (Deerwester et al., 1990) was one of the most influential works in this area. (Bengio et al., 2003) introduced the first neural language model which jointly learned word embeddings. Although such a language model was outperforming the baselines, it was not practical because of long training time requirements. (Collobert and Weston, 2008) proposed new neural architectures for word embeddings and showed that pre-trained word embeddings can be very valuable for some downstream NLP tasks. Word2Vec (Mikolov et al., 2013b,a) got very popular both because of its effectiveness and its ability to train models on very large text corpora efficiently. (Levy and Goldberg, 2014) showed that Word2Vec's skip-gram with negative sampling model (SGNS) is implicitly equivalent to word co-occurrence matrix factorization. Besides neural approaches, (Pennington et al., 2014) proposed an SVD based architecture which gained a lot of attention because it allowed to effectively consider the popularity of each word in the model definition.

FastText (Bojanowski et al., 2017) is a popular, recent proposal in the area of word embeddings. FastText introduces subword-level features to the Word2Vec framework which enables building embeddings for OOV words (see details in Section 3.1). An alternative approach, also capable of yielding representations for OOV words, is MIMIK (Pinter et al., 2017). MIMICK learns a function from input words to embeddings by minimizing the distance between embeddings produced by a char-based approach and the pretrained embeddings. As opposed to MOE, MIMICK does not support misspellings explicitly and it requires a set of pre-trained embeddings as input. We consider MIMICK to be a viable alternative to FastText which deserves future work exploring its performance on misspelled text.

\section{Misspelling Oblivious Embeddings}

\subsection{The FastText Model}

Our current work can be viewed as a generalization of FastText, which, in turn, extends the the skip-gram with negative sampling (SGNS) architecture, proposed as a part of the Word2 Vec framework. In this section we will briefly discuss major additions to SGNS introduded by FastText.

Let $V$ be a vocabulary of words and $T=$ $w_{1}, w_{2}, \ldots, w_{|T|}$ be a text corpus, represented as a sequence of words from $V$. We define the context of a word $w_{i} \in V$ as $C_{i}=$ $\left\{w_{i-l}, \ldots, w_{i-1}, w_{i+1}, \ldots, w_{i+l}\right\}$ for some $l$ set as a hyperparameter. In the SGNS model, a word $w_{i}$ is represented by a single embedding vector $\mathbf{v}_{i}$ equivalent to the input vector of a simple feedforward neural network, trained by optimizing the following loss function:

$$
\begin{array}{r}
L_{W 2 V}:=\sum_{i=1}^{|T|} \sum_{w_{c} \in C_{i}}\left[\ell\left(s\left(w_{i}, w_{c}\right)\right)+\right. \\
\left.\sum_{w_{n} \in N_{i, c}} \ell\left(-s\left(w_{i}, w_{n}\right)\right)\right]
\end{array}
$$

where $\ell$ denotes the logistic loss function $\ell(x)=$ $\log \left(1+e^{-x}\right)$ and $N_{i, c}$ is a set of negative samples drawn for the current word $w_{i}$ and its context $w_{c} \in$ $C_{i} . s$ is the scoring function, which for SGNS is defined as the the dot product $\mathbf{v}_{i}^{T} \mathbf{u}_{c}$, where $\mathbf{u}_{c}$ is an output vector associated with the word $w_{c}$ and $\mathbf{v}_{i}$ is an input vector associated with the word $w_{i}$. Therefore, $s\left(w_{i}, w_{c}\right)=\mathbf{v}_{i}^{T} \mathbf{u}_{c}$.

In FastText, we additionally embed subwords (also referred to as character $n$-grams) and use them to construct the final representation of $w_{i}$. Formally, given hyperparameters $m$ and $M$ denoting a minimum and a maximum length of an $n$-gram respectively, the FastText model embeds all possible character $n$-grams of the word such that $m \leq n \leq M$. E.g. given $m=3, M=5$ and the word banana, the set of $n$-grams we consider is ban, ana, nan, bana, anan, nana, banan, anana. Now, let $\mathcal{G}_{w_{i}}$ denote the set of all $n$-grams of a word $w_{i} \in V$ plus the word itself (e.g. $\mathcal{G}_{\text {banana }}$ is the set defined in the example above plus the word banana itself). Given $\mathcal{G}_{w_{i}}$, FastText scoring function for a word $w_{i}$ and a context $w_{c}$ is defined as follows:

$$
s_{F T}\left(w_{i}, w_{c}\right):=\sum_{\mathbf{v}_{g}, g \in \mathcal{G}_{w_{i}}} \mathbf{v}_{g}^{T} \mathbf{u}_{c}
$$

Therefore, the representation of $w_{i}$ is expressed through the sum of the representations of each of 
the $n$-grams derived from $w_{i}$ plus the representation of $w_{i}$ itself. FastText optimizes the loss function in Eq.1, but uses the scoring function $s_{F T}$ defined in Eq.2. An extensive experimentation has shown that FastText improves over the original Word2Vec skip-gram model. The loss function of FastText will be referred to as $L_{F T}$ throughout the rest of this work.

\subsection{The MOE Model}

As was shown empirically in the FastText paper, the $n$-grams which impact the final representation of a word the most in FastText correspond to morphemes. Based on this observation, we hypothesize that although FastText can capture morphological aspects of text, it may not be particularly resistant to misspellings which can occur also withing the dominant morphemes. In this section, we present the architecture of our model - MOE or Misspelling Oblivious (word) Embeddings. MOE holds the fundamental properties of FastText and Word2Vec while giving explicit importance to misspelled words.

Loss Function. The loss function of MOE is a weighted sum of two loss functions: $L_{F T}$ and $L_{S C} . L_{F T}$ is the loss function of FastText which captures semantic relationships between words. $L_{S C}$ or the spell correction loss aims to map embeddings of misspelled words close to the embeddings of their correctly spelled variants in the vector space. We define $L_{S C}$ as follows:

$$
\begin{array}{r}
L_{S C}:=\sum_{\left(w_{m}, w_{e}\right) \in M}\left[\ell\left(\hat{s}\left(w_{m}, w_{e}\right)\right)+\right. \\
\left.\sum_{w_{n} \in N_{m, e}} \ell\left(-\hat{s}\left(w_{m}, w_{n}\right)\right)\right]
\end{array}
$$

where $M$ is a set of pairs of words $\left(w_{m}, w_{e}\right)$ such that $w_{e} \in V$ is the expected (correctly spelled) word and $w_{m}$ is its misspelling. $N_{m, e}$ is a set of random negative samples from $V \backslash\left\{w_{m}, w_{e}\right\}$. $L_{S C}$ makes use of the logistic function $\ell(x)=$ $\log \left(1+e^{-x}\right)$ introduced in Section 3.1. The scoring function $\hat{s}$ is defined as follows:

$$
\hat{s}\left(w_{m}, w_{e}\right)=\sum_{\mathbf{v}_{g}, g \in \hat{\mathcal{G}}_{w_{m}}} \mathbf{v}_{g}^{T} \mathbf{v}_{e}
$$

where $\hat{\mathcal{G}}_{w_{m}}:=\mathcal{G}_{w_{m}} \backslash\left\{w_{m}\right\}$. Therefore, the scoring function is defined as the dot product between the sum of input vectors of the subwords of $w_{m}$ and the input vector of $w_{e}$. Formally, the term $\ell\left(\hat{s}\left(w_{m}, w_{e}\right)\right)$ enforces predictability of $w_{e}$ given $w_{m}$. Intuitively, optimizing $L_{S C}$ pushes the representation of a misspelling $w_{m}$ closer to the representation of the expected word $w_{e}$. It is also worth mentioning that embeddings for $w_{m}$ and $w_{e}$ share the same parameters set. The complete loss function of MOE, $L_{M O E}$, is defined as follows:

$$
L_{M O E}:=(1-\alpha) L_{F T}+\alpha \frac{|T|}{|M|} L_{S C}
$$

Optimizing the loss functions $L_{F T}$ and $L_{S C}$ concurrently is not a straightforward task. This is because two different loss functions iterate over two different datasets: the text corpus $T$, and the misspellings dataset $M$. The optimization process should be agnostic to the sizes of $T$ and $M$ in order to prevent results from being severely affected by those sizes. Therefore, we scale $L_{S C}$ with the coefficient $|T| /|M|$. This way the importance of a single Stochastic Gradient Descent (SGD) update for $L_{F T}$ becomes equivalent to a single SGD update for $L_{S C}$. Moreover, $\alpha$ is the hyperparameter which sets the importance of the spell correction loss $L_{S C}$ with respect to $L_{F T}$ thus making MOE a generalization of FastText.

\section{Data}

As mentioned in Section 3.2, MOE jointly optimizes two loss functions, each of which iterates over a separate dataset - a corpus of text for the FastText loss $L_{F T}$ and a set of pairs (misspelling, correction) for the spell correction loss $L_{S C}$. In this section, we will briefly discuss how we obtain each of these datasets.

\subsection{English text corpus}

We use an English Wikipedia dump ${ }^{2}$ as the text corpus $T$ to optimize $L_{F T}$. The baseline FastText model is also trained on this dataset. Matt Mahoney's perl script ${ }^{3}$ is used for pre-processing the raw Wikipedia dump. After pre-processing, the training corpus consists of $|T|=4,341,233,424$ words. When generating the vocabulary $V$ based on the corpus, we apply a frequency threshold of 5 . After deduplication and thresholding, the size of the vocabulary for our corpus is $|V|=2,746,061$ words. We also apply progressive subsampling of

\footnotetext{
${ }^{2}$ dumps.wikimedia.org

${ }^{3}$ http: / / mattmahoney $\cdot$ net/dc/textdata
} 
frequent words in order to not assign too much importance to highly frequent words.

\subsection{Misspelled data generation}

The misspellings dataset $M$ consists of a set of pairs $\left(w_{m}, w_{e}\right)$, where $w_{e} \in V$ represents a (presumably correctly spelled) word from the vocabulary and $w_{m}$ is a misspelling of $w_{e}$. Given the size of $V$, we opt for generating misspellings in an automated fashion, using an in-house misspellings generation script. The script is based on a simple error model, which captures the probability of typing a character $p_{m}$ when a character $p_{e}$ is expected (note that it's possible to have $p_{m}==p_{e}$ ), given a context of previously typed characters. The script is capable of generating misspellings of targeted edit distance for an input word $w_{i}$. In the reminder of this section, we'll discuss details of the script implementation.

Error model. In order to create the error model, we first mine query logs of a popular search engine $^{4}$ and identify cases where a query was manually corrected by a searcher. We then pivot on the modified character and for each such modification we save a triplet $\left(c, p_{m}, p_{e}\right)$, where $p_{m}$ is the pivot character before modification, $p_{e}$ is the the target character after modification and $c$ represents up to 3 characters preceding the pivot in the original query. E.g. given a query hello worjd corrected to hello world, we would generate four triplets: $[(w o r, j, l),($ or $, j, l),(r, j, l),(\epsilon, j, l))]$, where $\epsilon$ represents an empty word. Similarly, we create triplets by pivoting on characters which have not been modified. After processing all available logs, we count each unique triplet. For each unique pair $\left(c, p_{m}\right)$ of a context and a pivot, we then create a target list consisting of all possible targets $p_{e}$, each associated with its probability calculated based on counts. We then sort each target list in the order of decreasing probability.

Injecting misspellings. Let's consider a word $w_{i} \in V$ that we want to misspell. For each character $p \in w_{i}$, we take it's longest possible context $c$ (up to 3 characters) and we look up the target list corresponding to $(c, p)$. We then proceed along the target list, summing up the probabilities of subsequent targets until the sum is greater or equal to a randomly selected target probability $t p \in[0.0,1.0]$. We then choose the corresponding

\footnotetext{
${ }^{4}$ https: / / www. facebook. com
}

target $t$ as a replacement for $p$ (note that in the majority of the cases $t==p$ ). We repeat this process for every word from $V$.

In order to respect real distributions of words in the text corpus $T$, we set the number of misspellings generated for each word $w_{i} \in V$ to be equal to the square root of the number of appearances of $w_{i}$ in $T$. The total size of misspellings dataset generated in this fashion is $|M|=20,068,964$ pairs. We make the dataset of misspellings publicly available at https:// bitbucket.org/bedizel/moe.

\section{Experiments}

In this section, we describe the experimental set up used for training our models and the experiments we conducted.

\subsection{Experimental setup}

We use FastText ${ }^{5}$ as a baseline for comparison since it can generate embeddings for OOV words which makes it potentially suitable for dealing with misspellings. We train the baseline model using the default hyperparameters provided by the authors. We consider character $n$-grams of lengths between $m=3$ and $M=6$, and we use 5 negative samples for each positive sample. Training MOE requires optimizing two loss functions $L_{F T}$ and $L_{S C}$ jointly. For optimizing $L_{F T}$, we use the same parameters as in the baseline. Additionally, to optimize $L_{S C}$, we experiment with 5 negative samples per positive sample. We sweep over a range of values for the coefficient combining the two losses: $\alpha \in$ $\{0.01,0.05,0.1,0.5,0.25,0.5,0.75,0.95,0.99\}$.

Both FastText and MOE are trained using Stochastic Gradient Descent with a linearly decaying learning rate for 5 epochs to learn vectors with 300 dimensions. We evaluate the performance of MOE on the following tasks: (intrinsic) Word Similarity, Word Analogy and Neighborhood Validity; (extrinsic) POS Tagging of English sentences.

We report the overlap between the misspellings seen at training time and misspellings present in tests in Table 1.

\subsection{Intrisic Evaluation}

We evaluate MOE on two classic intrinsic tasks, namely Word Similarity and Word Analogy and

\footnotetext{
${ }^{5}$ https: / / fasttext.cc/
} 


\begin{tabular}{c|c} 
Test set & \% of unseen \\
\hline WS353 $r=0.125$ & 25.05 \\
WS353 $r=0.250$ & 57.06 \\
WS353 $r=0.375$ & 64.37 \\
RareWord $r=0.125$ & 44.67 \\
RareWord $r=0.250$ & 70.18 \\
RareWord $r=0.375$ & 78.84 \\
Word Analogies & 50.71 \\
Neighborhood Similarity & 69.1
\end{tabular}

Table 1: Percentages of test misspellings unobserved at training time per test set. The $r$ parameter indicates variants of respective word similarity test sets.

on a novel intrinsic task evaluating the distance between vector embeddings of misspellings and their correctly spelled variants.

Word Similarity. In the word similarity task, we evaluate how well embeddings generated by MOE can capture the semantic similarity between two words. For this purpose, we use two datasets: (i) WS353 (Finkelstein et al., 2001), and (ii) Rare Words (RW) (Luong et al., 2013). Both datasets contain pairs of words $w_{a}$, and $w_{b}$ annotated with a real value in the range $[0,10]$ representing the degree of similarity between $w_{a}$ and $w_{b}$ as perceived by human judges.

In order to evaluate how resilient our method is to spelling errors, for each pair of words $\left(w_{a}, w_{b}\right)$ in the dataset, we provide a respective pair of misspellings $\left(m_{a}, m_{b}\right)$. The misspellings are mined from search query logs of a real-world online search service. When desired misspellings are not available in the logs, we synthetically generate them using the same script we used to generate the set $M$ (see Section 4 for details). We create 3 misspelled variants of both WS353 and RW datasets. In each variant we limit the ratio between the edit distance (Levenshtein, 1966) of the word and the misspelling $d_{e}\left(w_{i}, m_{i}\right)$ and the length of the word by a constant $r$, where $r \in\{0.125,0.250,0.375\}$, with $r=0$ representing the original dataset. More precisely for each $r$ we look for a misspelling which satisfies the following condition $d_{e}\left(w_{i}, m_{i}\right)=\left\lfloor r * \operatorname{len}\left(w_{i}\right)\right\rfloor$. Effectively, if a word is too short to satisfy the condition, we preserve the original word (then $w_{i}=m_{i}$ ). Histograms in Figure 1 show the actual distribution of edit distances and lengths of words. As expected, edit distance increases steeply with the increase of $r$ value. Edit distances are higher for the
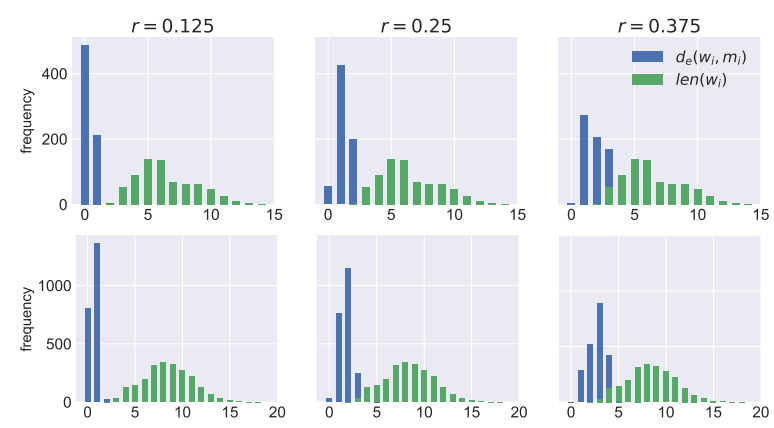

Figure 1: Distribution of edit distances $d_{e}\left(w_{i}, m_{i}\right)$ and lenghts of words $l e n\left(w_{i}\right)$ for WS353 variants (Top) and RW variants (Bottom).

RW dataset since in average the length of words in RW is higher than on average length of words in WS353. Also, we observe that for $r=0.125$, a significant portion of the words is not changed.

We conduct experiments for different values of the hyperparameter $\alpha$ which sets the trade-off between $L_{F T}$ and $L_{S C}$, i.e. the importance assigned to semantic loss and misspelling loss. In the experiments, results corresponding to $\alpha=0$ represents our baseline, FastText, since for $\alpha=0$ the loss $L_{M O E}$ is equal to $L_{F T}$.

We measure the Spearman's rank correlation (Spearman, 1904) between the distance of an input pair of words and the human judgment score both for the original and the misspelled pair. Figure 2 demonstrates the results of the word similarity task on the WS353 dataset. We observe that MOE is improving over FastText for WS353 variants with $r=0.25$, and $r=0.375$, and degrading performance when $r=0$, and $r=0.125$, where the majority of the words is not changed (see Figure 1 for the edit distance distribution). As we expected, larger values of $\alpha$, corresponding to more attention given to misspellings during training, result in improvements for highly misspelled datasets.

For the RW dataset (Figure 2), we observe that for all the values of $r$, MOE improves over the FastText baseline when we set $\alpha=0.05$. More specifically, when $r \in\{0,0.125\}$ and when $\alpha<\approx$ 0.1 , the proposed method improves over the baseline. When the amount of misspellings is higher, i.e., $r \in\{0.25,0.375\}$, MOE improves the results over the baseline for all of the $\alpha$ values. These results suggest that FastText may be a good baseline for dealing with low edit distance misspellings, however our model is better at capturing semantic relationships on higher edit distance misspellings. This is in line with our hypothesis presented in 

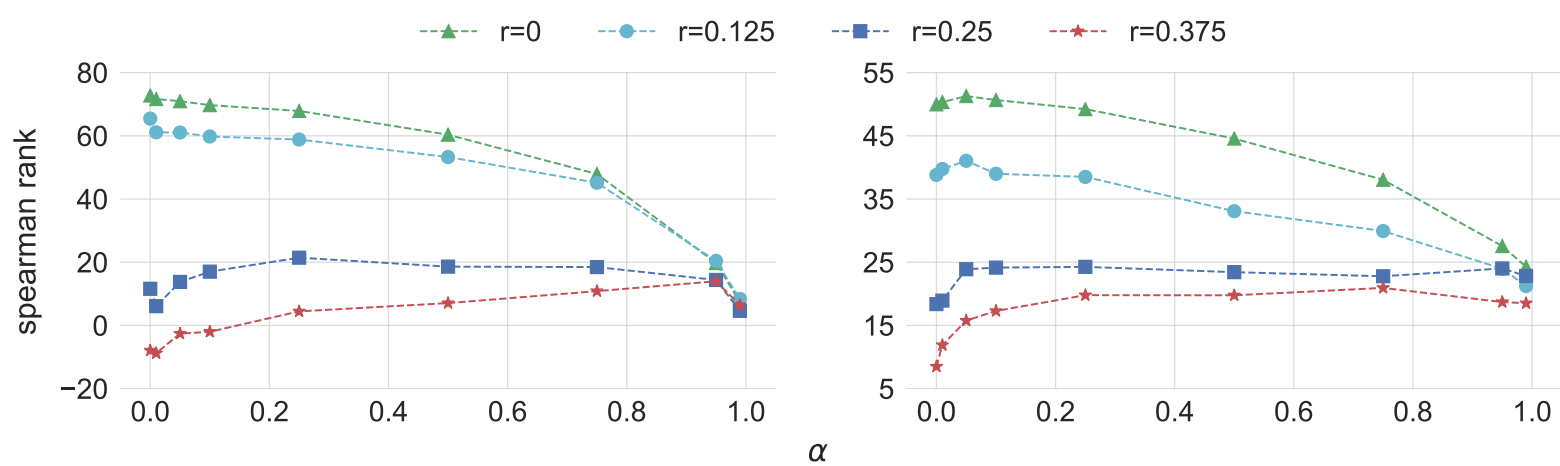

Figure 2: Experimental results for word similarity task for WS353 (Left) and RW (Right). $\alpha=0$ values represent our baseline, FastText.
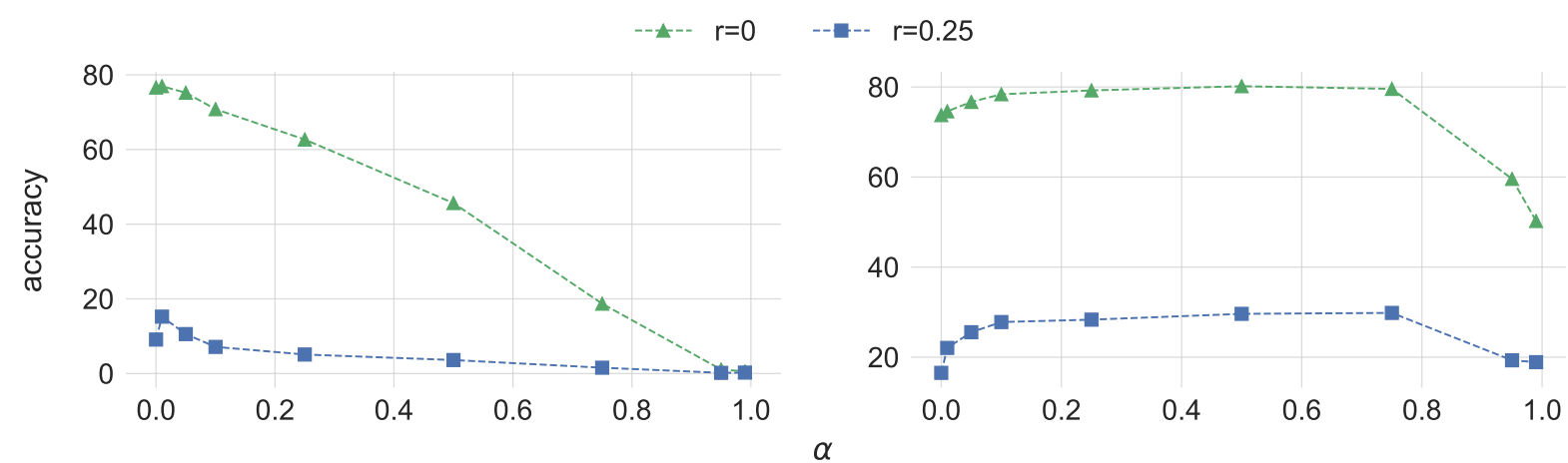

Figure 3: Experimental results for word analogy task, Semantic (Left) and Syntactic (Right). $\alpha=0$ values represent our baseline, FastText.

Section 3.2.

Word Analogy. In addition to the word similarity, we also test the performance of MOE on the popular word analogy task introduced by (Mikolov et al., 2013a). This task attempts to measure how good the embeddings model is at preserving relationships between words. A single test sample from the word analogy dataset consists of four words $A, B, C, D$, forming two pairs - $A, B$ and $C, D$, remaining in analogous relationships (" $A$ is to $B$ like $C$ is to $D "$ ). There are two types of relationships: (i) syntactic, related to the structure of words; and (ii) semantic, related to their meanings. banana, bananas, cat, cats is an example of a syntactic test sample. In both pairs the fact that the second word is a plural version of the first constitutes a relationship between them. Athens, Greece, Berlin, Germany is an example of a semantic test sample. The relationship which is being tested in this case is that between the capital of a country and the country itself.

In addition to analyzing the canonical variant of the word analogies test, we also introduce a mod- ification which is suitable specifically to the misspellings use-case. Given a line $A, B, C, D$ from the original analogies dataset, we misspell the first pair of words, obtaining a line $A^{\prime}, B^{\prime}, C, D$, where $A^{\prime}$ is a misspelling of $A$ and $B^{\prime}$ is a misspelling of $B$. We want to test if the misspelled pair $A^{\prime}, B^{\prime}$ preserves the relationship of the pair $C, D$. When generating misspellings we use a procedure similar to the one used for word similarities. We create one variant of the misspelled dataset, constraining the edit distance to $r=0.25$.

Experimental results for the canonical version of the word analogy task, presented in Figure 3, show that MOE performs worse than FastText on the semantic analogy task. On the other hand, MOE performs better than the baseline on the syntactic analogies task. The results for the misspelled variant of the task show that, the overall performance of both the baseline and MOE is worse than on the canonical variant. For low values of $\alpha \in\{0.01,0.05\}$, MOE outperforms the baseline on the semantic task, achieving an over $67 \%$ better score than FastText for $\alpha=0.01$. MOE outperforms the baseline on the syntactic 
task for all tested values of $\alpha$, improving by over $80 \%$ for $\alpha=0.75$. For $\alpha=0.01$, which achieved the best semantic result, the improvement on the syntactic task is over $33 \%$.

The trends that we observe both in the canonical and the misspelled variant of the word analogies task seem to validate our choice of the loss function for the MOE model. It is clear that the FastText component of the loss is indispensable to learn the semantic relationships between words. In fact, it is the only component of the loss function which attempts to learn these relationships. Therefore, decreasing it's importance (by increasing the value of $\alpha$ ) is reflected by a decay in the semantic analogies score. The spellcorrection component of the loss function, on the other hand, leverages the relationship between correctly spelled words and their misspellings. As a side effect, it also adds additional subword information into the model. This explains our good performance on the syntactic analogies task. As our results on the misspelled variant of the task show, we improve over the baseline in understanding analogies on the misspelled words, which was one of the design principles for MOE.

Neighborhood Validity. One of the explicit objectives of MOE is to embed misspellings close to their correct variants in the vector space. In order to validate this hypothesis, we check where in the neighborhood of a misspelling the correct word is situated. Formally, for a pair $\left(w_{m}, w_{e}\right)$ of a misspelling and its correction, we pick $k$ nearest neighbors of the misspelling $w_{m}$ in the embedding space using cosine similarity as a distance metric. We then evaluate the position of the correct word $w_{e}$ within the neighborhood of $w_{m}$ using two metrics:

- We use MRR (Voorhees et al., 1999) to score the neighborhood of the embeddings of misspellings (we assign a score of 0 if the correct word is not present).

- We also compute the neighborhood coverage defined as the percentage of misspellings for which the neighborhood contains the correct version.

The test set contains 5,910 pairs $\left(w_{m}, w_{e}\right)$ sampled from a collection of data coming from a realworld online service ${ }^{6}$. Figure 4 shows experimen-

\footnotetext{
${ }^{6}$ WWw . facebook. com
}

tal results for Neighbor Validity task. We remind that $\alpha=0$ denotes the FastText baseline.

The test results confirm our hypothesis. We observe that MRR increases when more importance is given to the $L_{S C}$ component of the loss for any size of the neighborhood $k \in\{5,10,50,100\}$. A similar trend can be observed for the neighborhood coverage task. We conclude that, on average, we're more likely to surface the correction using MOE than with FastText. What is more, whenever we are able to surface the correct version of a misspelled word, its position in the ranking is higher for MOE than for the FastText baseline.

\subsection{Extrinsic Evaluation}

POS Tagging. Finally, we evaluate MOE on a Part-of-Speech (POS) tagging task ${ }^{7}$. To assess the impact of misspellings we artificially inject misspellings in the dataset. We train MOE on three different dataset variants: a non-misspelled dataset, to verify that MOE does not jeopardize the performance on correct words; a dataset where $10 \%$ of words contain a misspelling, to simulate a realistic environment where some of the words are misspelled; and finally on a dataset where $100 \%$ of words contain misspellings, to simulate a highly distorted environment. We use a state-of-the-art POS tagger (Ma and Hovy, 2016) consisting of a Conditional Random Fields (CRF) model where embeddings of the words in a sentence constitute observations and the tags to assign constitute the latent variables. This model adds a dependency on both layers of a Bi-LSTM component to the tag variables in the CRF. We evaluate the F1 score of the system for the three dataset variants we describe above. We test two different representations as input to the CRF: FastText (our baseline), and MOE embeddings. Our results are reported in Table 2 .

We make the following observations based on the results of our experiments. Firstly, in the two extreme cases of the $100 \%$ misspelled test and correct training and the correct test and $100 \%$ misspelled training, MOE improves the F1 by 2 and 3.5 points respectively with respect to the FastText baseline. When the test data is $100 \%$ misspelled, MOE always beats the baseline by up to 2.3 points of F1. Also, in this case the loss in F1 with respect to the case where both the training and the test are

\footnotetext{
${ }^{7}$ http: / / universaldependencies.org/ conl117/data.html
} 


\begin{tabular}{c|c|c|c|c|c|c} 
Test Data & \multicolumn{3}{|c|}{$100 \%$ Misspelled } & \multicolumn{3}{c}{ Original } \\
\hline Training Data & Original & $100 \%$ Miss. & $10 \%$ Miss. & Original & $100 \%$ Miss. & $10 \%$ Miss. \\
\hline FastText, $\alpha=0.0$ & 30.47 & 79.71 & 65.70 & 94.33 & 57.16 & 94.14 \\
MOE, $\alpha=0.01$ & 29.04 & 80.66 & 67.94 & $\mathbf{9 4 . 5 5}$ & 59.11 & 94.21 \\
MOE, $\alpha=0.05$ & 28.52 & $\mathbf{8 1 . 1 7}$ & $\mathbf{6 8 . 9 2}$ & 94.25 & 58.95 & 93.92 \\
MOE, $\alpha=0.1$ & 30.94 & 80.97 & 67.30 & 94.45 & 58.88 & $\mathbf{9 4 . 2 9}$ \\
MOE, $\alpha=0.25$ & 29.00 & 80.13 & 67.63 & 94.37 & 58.67 & 94.01 \\
MOE, $\alpha=0.5$ & 29.19 & 80.43 & 66.76 & 94.27 & 57.29 & 93.94 \\
MOE, $\alpha=0.75$ & 30.94 & 78.65 & 64.53 & 94.18 & 57.67 & 93.81 \\
MOE, $\alpha=0.95$ & 32.40 & 75.28 & 62.29 & 93.09 & 60.21 & 92.52 \\
MOE, $\alpha=0.99$ & $\mathbf{3 2 . 5 7}$ & 73.36 & 61.36 & 90.91 & $\mathbf{6 0 . 6 2}$ & 90.53 \\
\hline
\end{tabular}

Table 2: Performance on POS tagging task for UPOS tags using CRF. The models were trained on 100 epochs with an early stop (small difference on validation error) mechanism enabled. Considering F1 score, we evaluate on 2 variants of test data: Original (correctly spelled) on the right hand side of the table and $100 \%$ misspelled on the left hand side.

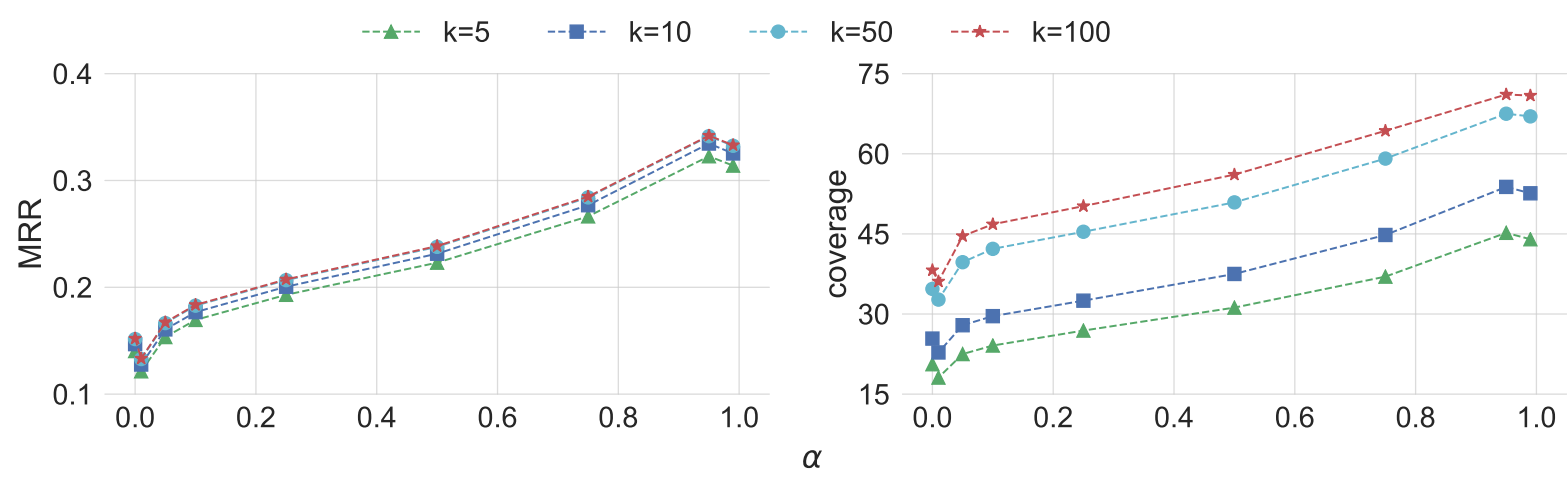

Figure 4: Experimental results for the Neighborhood Validity task. $\alpha=0$ values represent our baseline, FastText. On the left hand side we present the resulting MRR scores. On the right hand size we present the results for the neighborhood coverage.

correct is much less then when the training data does not contain misspellings. To be remarked is the F1 score difference in the more realistic case consisting of training data that is $10 \%$ misspelled. In this case MOE attains a sensitive improvement of $2.3 \%$ points of F1. Finally, MOE does not reduce the effectiveness of the CRF POS Tagger with respect to the FastText baseline when neither the training nor the test set are misspelled. All in all, we have shown that MOE does not affect the effectiveness of the POS Tagger in the case of correctly misspelled words and improves sensitively the quality of the POS tagger on misspellings.

\section{Conclusion and Future Work}

One of the most urgent issues of word embeddings is that they are often unable to deal with malformed words which is a big limitation in the real-world applications. In this work, we proposed a novel model called MOE, which aims to solve a long-standing problem: generating high quality, semantically valid embeddings for misspellings.

In the experiments section, in the neighborhood validity task, we show that MOE maps embeddings of misspellings close to embedding of the corresponding correctly spelled word. Moreover, we show that MOE is performing significantly better than the FastText baseline for the word similarity task when misspellings are involved. For the canonical versions of the word similarity tasks, where misspellings are not involved, we show that MOE doesn't worsen the quality significantly for the WS353 dataset and improves over baseline for the RW dataset. In the word analogy task, MOE is able to preserve the quality of the semantic analogies similar to the baseline, while improving on the syntactic analogies. In the variant of the test where misspellings are involved, MOE outperforms the baseline on both semantic and syntactic questions. Finally, we have shown that MOE does 
not affect the effectiveness of the POS Tagger in the case of correctly spelled words and improves sensitively the quality of the POS tagger on misspellings.

In the future, we will test different ways of training embeddings for misspellings including the extension of the same technique to multi-lingual embeddings. We are going to test deep architectures to combine the $n$-grams in misspellings to better capture various interdependencies of $n$-grams and correct versions of words. Finally, we will assess the robustness of both character-based (Kim et al., 2016) and context-dependent embeddings (Devlin et al., 2018), (Peters et al., 2018) with respect to misspellings.

\section{References}

Yoshua Bengio, Réjean Ducharme, Pascal Vincent, and Christian Jauvin. 2003. A neural probabilistic language model. Journal of machine learning research, 3(Feb):1137-1155.

Piotr Bojanowski, Edouard Grave, Armand Joulin, and Tomas Mikolov. 2017. Enriching word vectors with subword information. Transactions of the Association for Computational Linguistics, 5:135-146.

Ronan Collobert and Jason Weston. 2008. A unified architecture for natural language processing: Deep neural networks with multitask learning. In Proceedings of the 25th international conference on Machine learning, pages 160-167. ACM.

Silviu Cucerzan and Eric Brill. 2004. Spelling correction as an iterative process that exploits the collective knowledge of web users. In Proceedings of EMNLP 2004, pages 293-300.

Scott Deerwester, Susan T Dumais, George W Furnas, Thomas K Landauer, and Richard Harshman. 1990. Indexing by latent semantic analysis. Journal of the American society for information science, 41(6):391.

Jacob Devlin, Ming-Wei Chang, Kenton Lee, and Kristina Toutanova. 2018. Bert: Pre-training of deep bidirectional transformers for language understanding. arXiv preprint arXiv:1810.04805.

Lev Finkelstein, Evgeniy Gabrilovich, Yossi Matias, Ehud Rivlin, Zach Solan, Gadi Wolfman, and Eytan Ruppin. 2001. Placing search in context: The concept revisited. In Proceedings of the 10th international conference on World Wide Web, pages 406414. ACM.

Geoffrey E Hinton. 1986. Learning distributed representations of concepts. In Proceedings of the eighth annual conference of the cognitive science society, volume 1, page 12. Amherst, MA.
Yoon Kim, Yacine Jernite, David Sontag, and Alexander M Rush. 2016. Character-aware neural language models. In AAAI, pages 2741-2749.

Vladimir I Levenshtein. 1966. Binary codes capable of correcting deletions, insertions, and reversals. Soviet physics doklady, 10(8):707-710.

Omer Levy and Yoav Goldberg. 2014. Neural word embedding as implicit matrix factorization. In $A d$ vances in neural information processing systems, pages 2177-2185.

Thang Luong, Richard Socher, and Christopher Manning. 2013. Better word representations with recursive neural networks for morphology. In Proceedings of the Seventeenth Conference on Computational Natural Language Learning, pages 104-113.

Xuezhe Ma and Eduard Hovy. 2016. End-to-end sequence labeling via bi-directional lstm-cnns-crf. arXiv preprint arXiv:1603.01354.

Tomas Mikolov, Kai Chen, Greg Corrado, and Jeffrey Dean. 2013a. Efficient estimation of word representations in vector space. CoRR, abs/1301.3781.

Tomas Mikolov, Ilya Sutskever, Kai Chen, Greg S Corrado, and Jeff Dean. 2013b. Distributed representations of words and phrases and their compositionality. In Advances in neural information processing systems, pages 3111-3119.

Jeffrey Pennington, Richard Socher, and Christopher D Manning. 2014. Glove: Global vectors for word representation. In EMNLP, volume 14, pages 1532 1543.

Matthew E. Peters, Mark Neumann, Mohit Iyyer, Matt Gardner, Christopher Clark, Kenton Lee, and Luke Zettlemoyer. 2018. Deep contextualized word representations. In Proc. of NAACL.

Yuval Pinter, Robert Guthrie, and Jacob Eisenstein. 2017. Mimicking word embeddings using subword rnns. In Proceedings of the 2017 Conference on Empirical Methods in Natural Language Processing, pages 102-112.

Charles Spearman. 1904. The proof and measurement of association between two things. The American journal of psychology, 15(1):72-101.

Ellen M Voorhees et al. 1999. The trec-8 question answering track report. In Trec, volume 99, pages 77 82. 